\title{
Ambient seismic noise tomography of SW Iberia integrating seafloor- and land-based data
}

\author{
Carlos Corela (1), Graça Silveira (1,2), Luís Matias (1), Martin Schimmel (3), and Wolfram Geissler (4) \\ (1) IDL, FFCUL, VAT 503183504, Lisboa, Portugal (mdsilveira@fc.ul.pt), (2) Instituto Superior de Engenharia de Lisboa, \\ Lisboa, Portugal, (3) Instituto de Ciencias de la Tierra Jaume Almera, Barcelona, Spain, (4) Alfred Wegener Institute, \\ Bremerhaven, Germany
}

We used ambient seismic noise recorded by 24 broadband ocean bottom seismometers (OBS-BB) deployed in in the Gulf of Cadiz during the EC funded NEAREST project and seven broadband land stations located in the South of Portugal to image the sedimentary and crustal structure beneath the Eastern Atlantic and SW Iberia. We computed ambient noise cross-correlations to obtain empirical Green's functions (EGFs) between all station pairs, and using both sort of sensors, namely seismometers and hydrophones. Despite the great difference between the crustal structure below beneath OBSs and land stations and the recording conditions, we were able to compute high signal-to-noise ratio EGFs, by applying a linear cross-correlation with a running absolute mean average time normalization, followed by a time-frequency phase weighted stack.

Dispersion analysis was then applied to the EGFs, between 4 and 20s period. The obtained 395 reliable group velocity dispersion curves, between all station pairs, allowed mapping the lateral variation of Rayleigh wave group velocities, as a function of period. Finally, dispersion curves extracted from each cell of the 2D group velocity maps were inverted, as a function of depth, to obtain the 3D distribution of the shear-wave velocities.

The 3-D shear wave velocity model, computed from joint inversion of OBS and land stations data allowed to estimate the thickness of sediments and crust and the Moho depth.

Although, we could perceive the impact of the spatial gap between OBSs and land stations, our model displays a good correlation with the main geological features. The main results on the sedimentary layer thickness and on the Moho depth are in agreement with the model proposed by other studies using observations from multi-beam bathymetry and seismic profiling, thus confirming that, not only that ambient noise tomography is a valuable tool to image oceanic domains, but also that we can integrate seafloor- and land-based stations.

Publication supported by FCT through UID/GEO/50019/2013 - Instituto Dom Luiz. 